\title{
Les tarifs sous les feux de la politique de santé
}

\section{Patrick Müllera, Christian Oeschger ${ }^{b}$, Thomas Kessler $^{c}$}

${ }^{a} \mathrm{FMH}$, chef de division, division Médecine et tarifs ambulatoires; ${ }^{b} \mathrm{FMH}$, chef de projet, division Médecine et tarifs ambulatoires;

${ }^{c} \mathrm{FMH}$, expert, division Médecine et tarifs ambulatoires

Placée sous le thème "Les tarifs sous les feux de la politique de santé», la deuxième Journée des délégués tarifaires de l'année, qui s'est tenue le 28 septembre 2017 à Berne, a enregistré un nouveau record avec la présence de plus de 100 participants.

Le Dr Urs Stoffel a souhaité la bienvenue aux nombreux participants et ouvert la Journée des délégués tarifaires en faisant le point sur la situation en politique de santé et en donnant un aperçu du programme, des exposés et de la table ronde à venir.

U. Stoffel a commencé par évoquer l'évolution des coûts de la santé et ses causes, en expliquant les raisons pour lesquelles les tarifs ambulatoires sont précisément aujourd'hui au cœur des débats de la politique de santé.

Puis, le chef du département Médecine et tarifs ambulatoires a enchaîné avec le projet TARCO, qui souffle désormais sa première bougie, un an après l'adoption du concept général par l'Assemblée des délégués en septembre 2016. Certains objectifs-clés du projet ont d'ores et déjà pu être atteints suite à l'Assemblée des délégués de janvier 2017: la nomenclature est pratiquement terminée et les négociations sur les modèles de coûts avec les partenaires tarifaires ont débuté.

Vous trouverez tous les documents et la présentation PowerPoint de la Journée sur le site internet de la FMH: www.fmh.ch $\rightarrow$ Tarifs ambulatoires $\rightarrow$ Journée des délégués tarifaires $\rightarrow$ Septembre $2017 \rightarrow$ Présentation

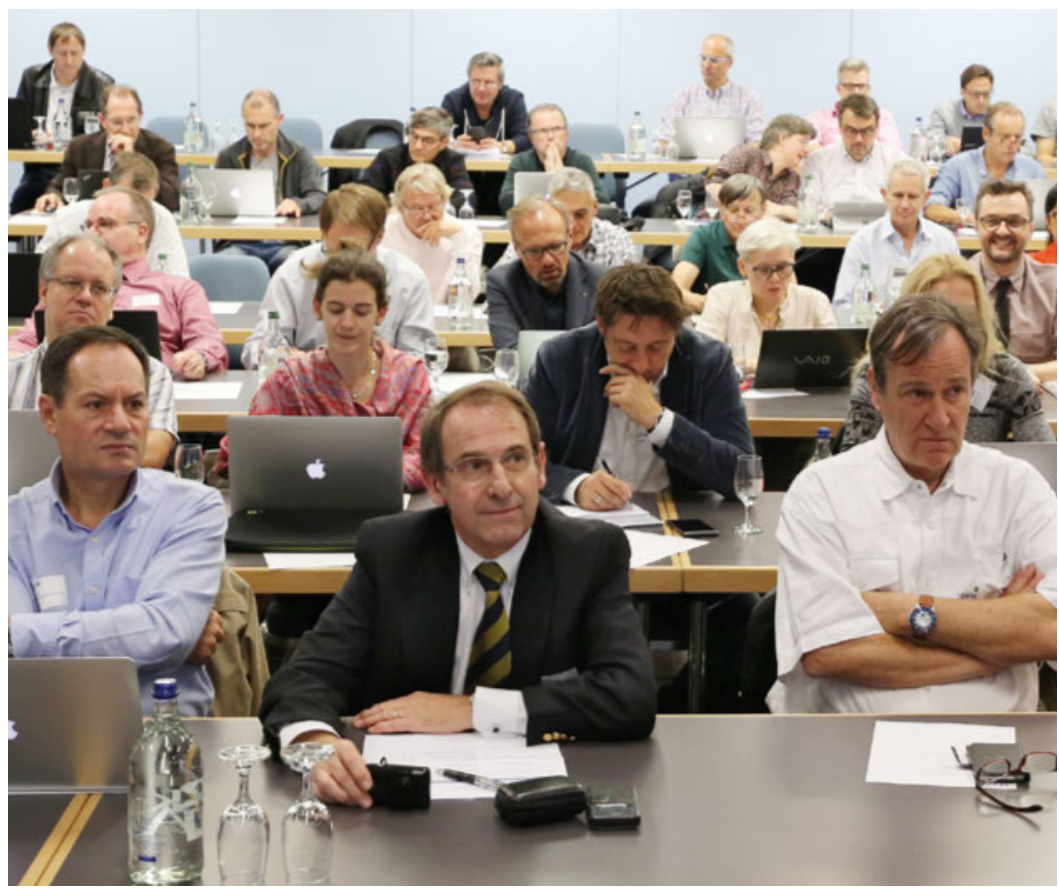

La Journée des délégués tarifaires s'est tenue dans les locaux de I'Hôtel Ador à Berne.

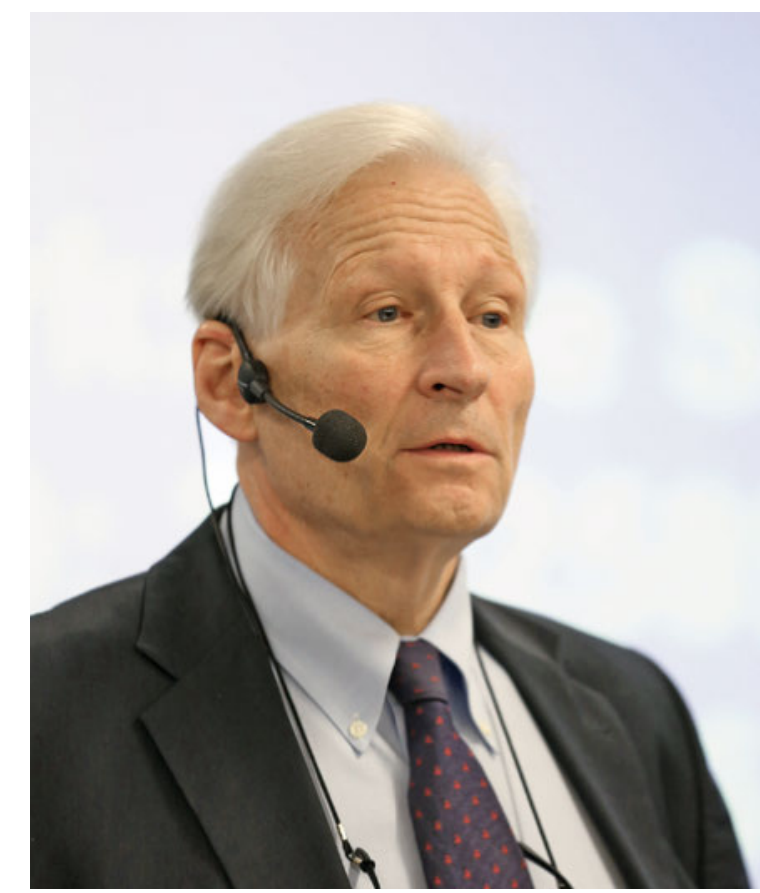

Le Dr Urs Stoffel souhaite la bienvenue aux nombreux participants. 


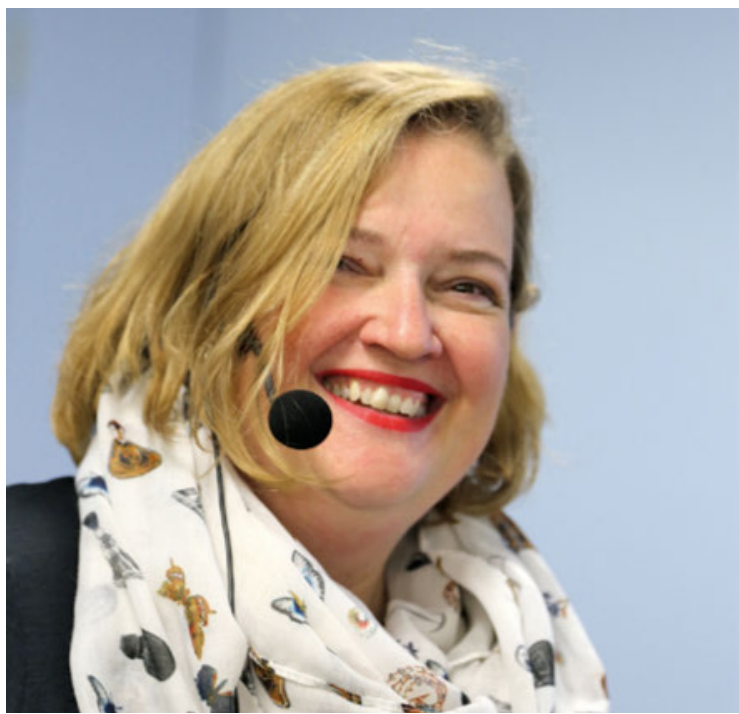

La Dresse Susanne Christen présente les derniers développements dans le domaine du laboratoire au cabinet.

\section{Nouveautés dans le domaine du laboratoire au cabinet}

La Dresse Susanne Christen a ensuite présenté les derniers développements dans le domaine du laboratoire au cabinet. Après 2009, la Liste des analyses fait actuellement l'objet d'une nouvelle révision (projet transAL) dans le but de supprimer les positions obsolètes et redondantes.

S. Christen en a profité pour évoquer la motion Hess (objet 16.3193) actuellement au Parlement. La motion prévoit de modifier l'article 52 de la loi fédérale sur l'assurance-maladie (LAMal) de manière à ce que les tarifs des analyses pratiquées par les laboratoires médicaux soient négociés par les partenaires tarifaires, sur le modèle de la structure tarifaire TARMED et des forfaits par cas DRG, ou, s'ils ne parviennent pas à s'entendre, que ces tarifs soient fixés par une autorité de recours instituée par les partenaires tarifaires. Sans oublier la motion Kuprecht (16.3487) qui demande l'introduction de la liberté de contracter pour les tarifs des laboratoires.

Enfin, S. Christen fait le point sur la situation actuelle de la Commission suisse pour l'assurance de qualité dans le laboratoire médical (QUALAB).

\section{Deuxième intervention du Conseil fédéral dans le TARMED}

Patrick Müller a commencé par donner quelques informations sur la deuxième intervention du Conseil fédéral dans le tarif TARMED et, faute d'informations de la part des autorités fédérales, est revenu sur les développements de ces dernières années et sur la compétence subsidiaire du Conseil fédéral. Les années de blocage dans le domaine des tarifs et l'échec de la révision ont mené à un élargissement des compétences du Conseil fédéral, lequel peut, depuis début 2013, intervenir activement dans la structure tarifaire en place par voie d'ordonnance. Ses compétences se limitent au domaine ambulatoire de la loi sur l'assurance-maladie (LAMal). Le 16 août 2017, le Conseil fédéral a rendu une décision de principe dans laquelle il maintient son intervention tarifaire au $1^{\mathrm{er}}$ janvier 2018. Suite aux nombreuses prises de position reçues lors de la consultation (plus de 600), principalement de la part des médecins et des sociétés de discipline médicale, il a toutefois apporté quelques corrections à son projet.

Pour P. Müller, il est évident qu'avec son intervention tarifaire, le Conseil fédéral compromet sa propre stratégie en matière de santé, "Santé2020", de même que de nombreux programmes fédéraux comme la stratégie en matière de soins palliatifs, la stratégie MNT, la stratégie en matière de démence et la stratégie Addictions. Dans l'ensemble, cette nouvelle intervention tarifaire, qui n'est ni appropriée ni conforme aux règles de l'économie d'entreprise, affaiblira considérablement la médecine ambulatoire et n'incitera aucunement à fournir les prestations dans le domaine ambulatoire, pourtant nettement moins cher et plus efficace.

\section{Projet TARCO - dernières informations}

Sabine Zehnder et Christian Oeschger ont quant à eux fait le point sur le projet TARCO. Organe de pilotage et de décision, le "cockpit» se compose de représentants de toutes les organisations faîtières de la FMH, de représentants des cantons et de l'AMDHS. Jusqu'ici, il a

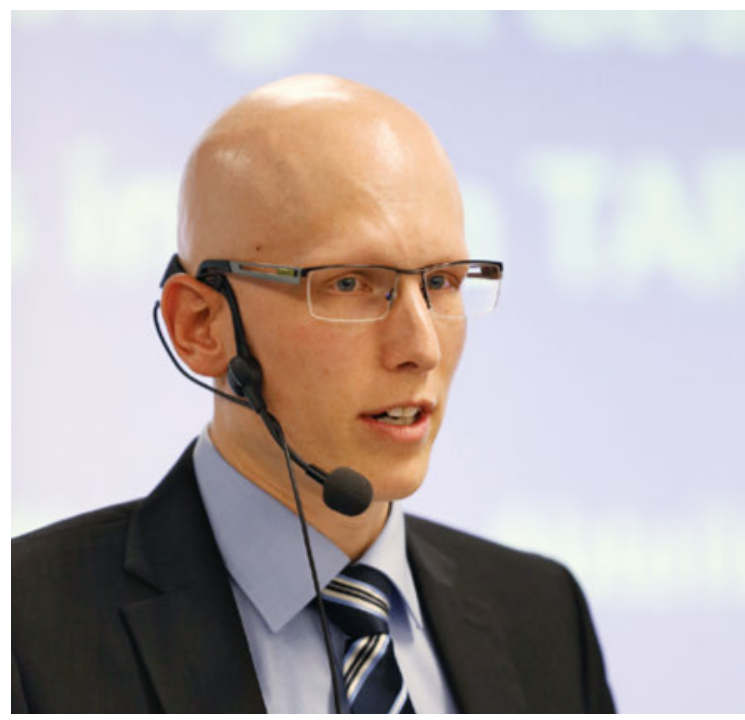

Patrick Müller donne des informations sur la deuxième intervention du Conseil fédéral dans le tarif TARMED. 

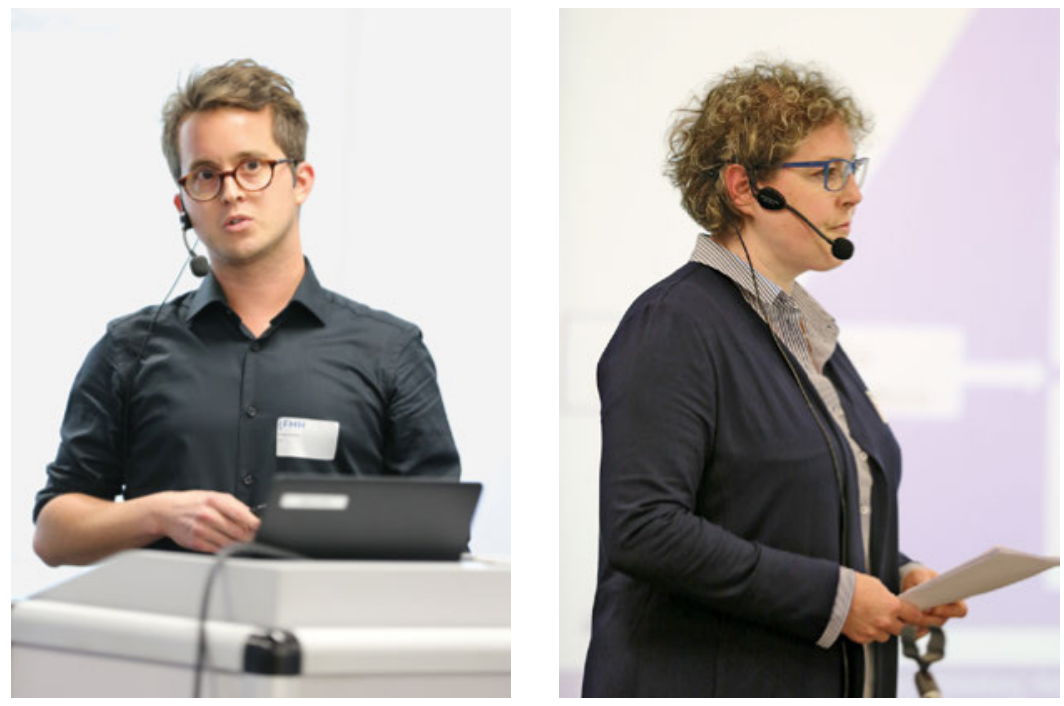

Christian Oeschger et Sabine Zehnder présentent les travaux actuels dans le cadre du projet TARCO. tions de traitement et de conseil, le contrôle de la plausibilité des prestations à l'acte et les groupes de travail «Valeurs intrinsèques» et «OP».

\section{Les partenaires tarifaires en route vers une structure tarifaire commune: illusion ou réalité?}

La table ronde animée par le Prof. Urs Brügger de l'Institut d'économie de la santé de Winterthour a été l'occasion d'entendre des personnalités de renom: Pius Zängerle (directeur curafutura), Verena Nold (directrice santésuisse), le Dr Urs Stoffel (Comité central de la FMH), Josef Müller (membre du Comité directeur de $\mathrm{H}+$ ), le Dr Josef Brandenberg (président $\mathrm{fmCh}$ ) et le Dr Philippe Luchsinger (président mfe) ont débattu des attentes et de la réalité en lien avec la révision du tarif médical ambulatoire.

Pour débuter, le Prof. Brügger a demandé aux participants au débat d'indiquer quelle était leur vision concernant le tarif ambulatoire, et de lui décrire quel pourrait être le titre des journaux le jour suivant l'adoption et le dépôt d'une structure tarifaire conjointe par tous les partenaires. Tous les participants à la table ronde se sont montrés plus ou moins d'accord sur l'importance de poursuivre le développement de la structure tarifaire et la nécessité pour les cinq partenaires de trouver un compromis. La une des journaux pourrait donc être: «Les partenaires tarifaires ont trouvé un accord

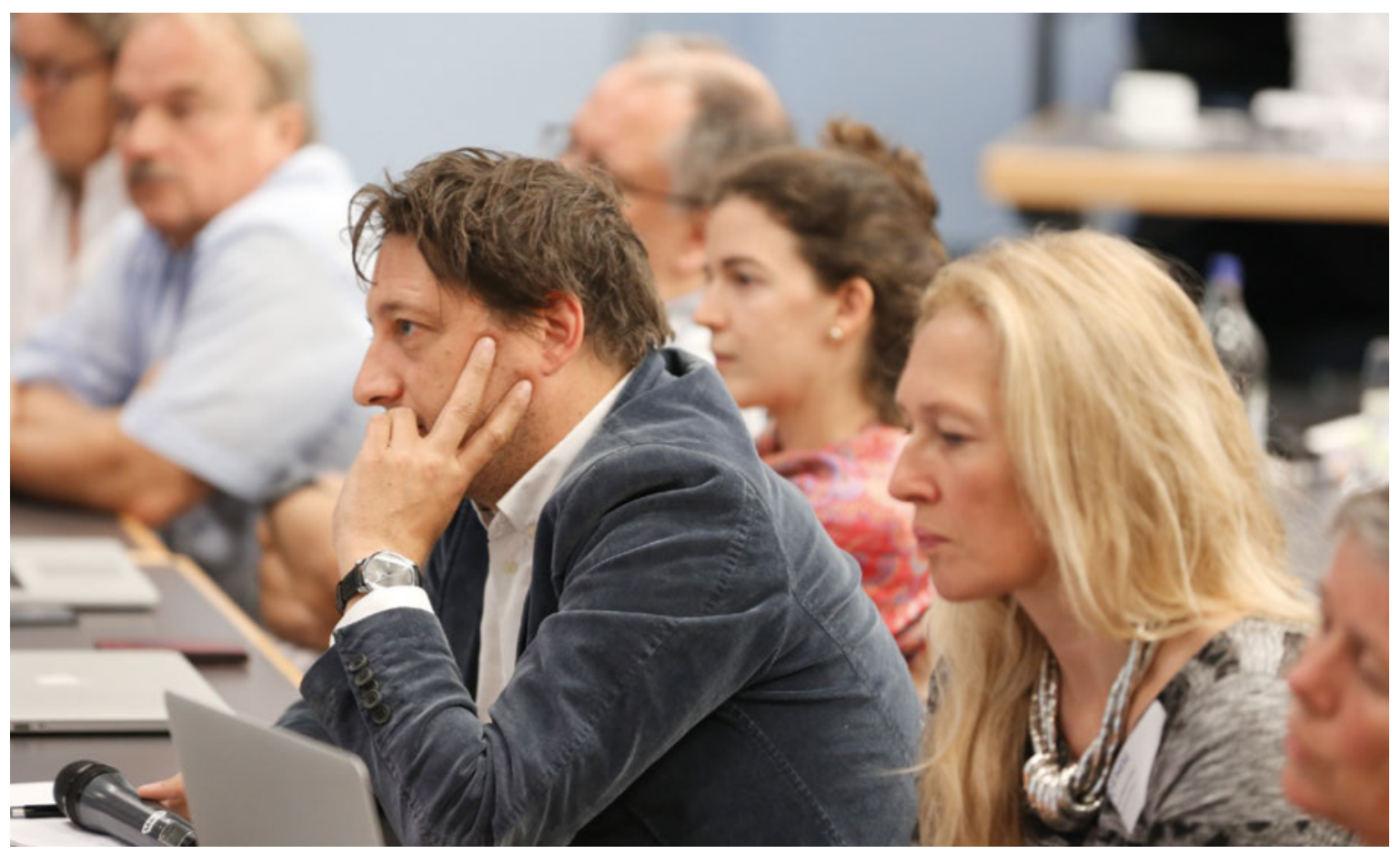

Les exposés ont suscité un vif intérêt de la part des participants à la Journée des délégués tarifaires. 


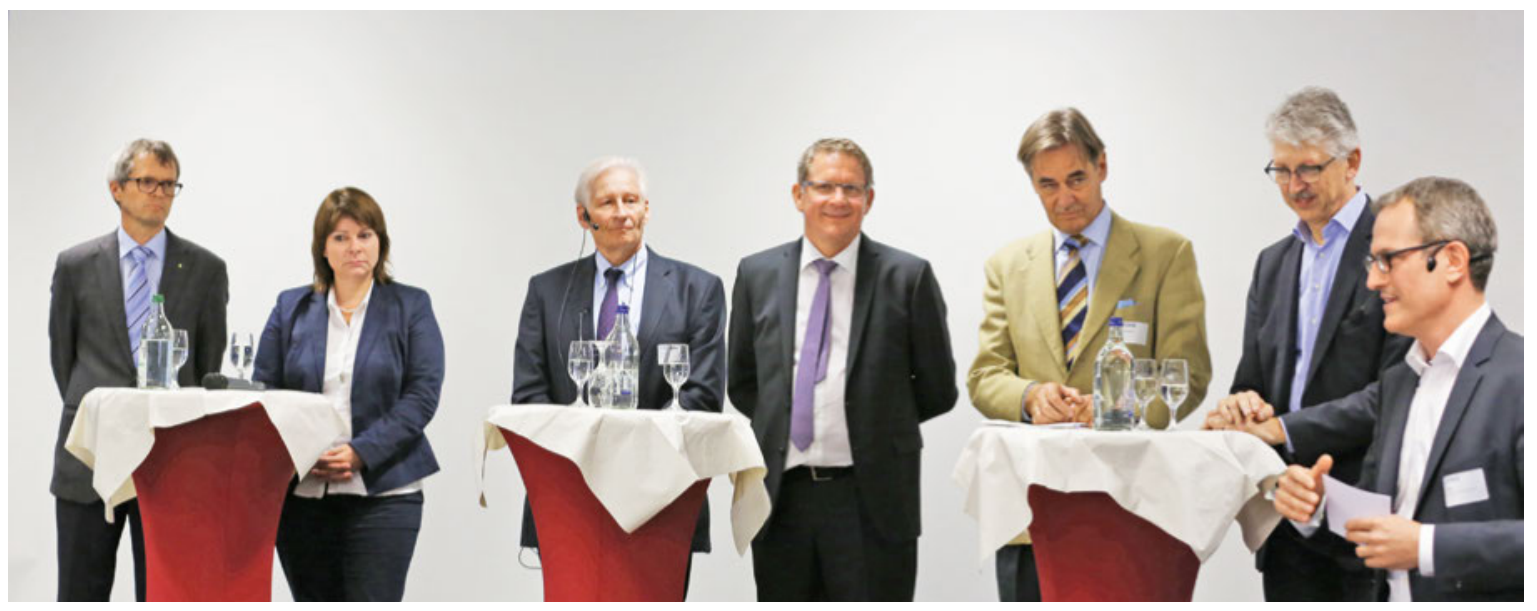

Le Prof. Brügger anime le débat.

sur le tarif TARMED.» Pour les participants, il est également important de mettre sur pied une organisation commune aux partenaires tarifaires chargée du suivi du tarif, sur le modèle de SwissDRG SA. S'agissant du mode de tarification des prestations, les vues divergent entre ceux qui désirent un tarif à la prestation détaillé et ceux qui souhaitent davantage de forfaits afin que le tarif contienne moins de prestations. Finalement, il est clair pour tous que les partenaires tarifaires doivent être prêts à faire des concessions et des compromis afin d'atteindre ensemble l'objectif visant «un nouveau tarif».

Correspondance: FMH, division Médecine et tarifs ambulatoires Baslerstrasse 47 CH-4600 Olten Tél. 0313591230 Fax 0313591238 tarife.ambulant[at]fmh.ch

\section{Rétrospective et perspective}

Pour conclure, le Dr Urs Stoffel dresse un bilan et formule le constat suivant: la pression politique va continuer de croître. Avec l'augmentation des primes, la population deviendra de plus en plus sensible et de moins en moins patiente. Le projet TARCO est peutêtre la dernière chance de révision globale et conjointe du TARMED avec tous les partenaires. U. Stoffel est convaincu que le corps médical doit absolument saisir cette chance et prouver qu'il a la volonté de maintenir un partenariat tarifaire actif. Cela suppose toutefois que toutes les parties soient prêtes à faire des compromis. "Serrons donc les rangs en direction d'un tarif négocié par tous afin d'éviter un tarif imposé par l'Etat!»

\section{Crédit photos}

Andreas Weissenburger

La prochaine Journée des délégués tarifaire aura lieu le jeud 26 avril 2018 à I'Hôtel Bern à Berne. Notez d'ores et déjà cette date dans votre agenda! 